\title{
Astronomical Organ Music
}

\section{Matthew Whitehouse}

\begin{abstract}
Music inspired by astronomical phenomena is familiar to INSAP participants. In this presentation, I will present two pieces of astronomy-inspired music composed specifically for the organ: Nebulae and Nova. The music will be accompanied by a slide show of astronomical images. Nebulae is my original work and is a musical narrative on the process of star formation. It has been performed throughout the United States, including at Notre Dame Cathedral in Paris in October 2010. Nova is by the American composer Myron Roberts, and is a depiction of a supernova explosion.
\end{abstract}

Music inspired by astronomical concepts and phenomena is familiar to INSAP participants. The author's presentation at INSAP VII featured two works for solo organ inspired by astronomical phenomena: his original composition Nebulae (2008), and Nova (1973) by American composer Myron Roberts. ${ }^{1}$ Nebulae is a musical narrative on the process of star formation, and Nova is a musical evocation of a supernova explosion.

The pipe organ, while normally associated with the church, is an ideal medium for astronomically-inspired music. The dynamic range of the organ is exceptionally large; it is capable of being both very powerful and very quiet and can switch between these two extremes very quickly. Additionally, the many sets of pipes in an organ make the instrument capable of numerous tone colours, and these colours can be combined to create an almost unlimited tonal palette. Furthermore, the electric blowers that drive modern pipe organs produce a steady and uninterrupted stream of wind, allowing sonorities to be sustained indefinitely. Because of these capabilities, the organ is ideally suited to music of great power, mystery, color, and spaciousness - qualities found in many compositions inspired by astronomical phenomena. Before we consider the two compositions mentioned above, a few words about my background are in order. I am an organist and composer, and graduated with a Doctor of Musical Arts degree in organ performance at The University of Arizona in Tucson in

1 Matthew Whitehouse, Nebulae, Unpublished manuscript, 2008; Myron J. Roberts, Nova. Melville, NY: Belwin-Mills, 1973. Sound recordings of Nebulae and Nova can be found at, http://www.matthewwhitehouse.com.

Matthew Whitehouse, 'Astronomical Organ Music', eds. Nicholas Campion and Rolf Sinclair, Culture and Cosmos, Vol. 16 nos. 1 and 2, 2012, pp. 393-397. www.CultureAndCosmos.org 


\section{Astronomical Organ Music}

2012 (after this paper was delivered at the 2010 INSAP conference). In addition to my work as an organist and composer, I am active as an astronomy educator: I have served on the staff of Kitt Peak National Observatory's nightly public astronomy programmes, and am an instructor for the summer Astronomy Camps sponsored by The University of Arizona Department of Astronomy/Steward Observatory. ${ }^{2}$ I was also an astronomer volunteer for Project ASTRO-Tucson, an educational outreach programme sponsored by the Astronomical Society of the Pacific that pairs astronomers with classroom teachers to enhance astronomy and science instruction. ${ }^{3}$ Currently, I hold the position of Observatory Manager at the South Carolina State Museum in Columbia.

The first composition featured in the author's presentation at INSAP VII was his own Nebulae, a solo organ work of approximately eight minutes' duration. As mentioned earlier, Nebulae is a musical narrative on the process of star formation; each section of the piece corresponds to one of the stages of starbirth as currently understood by astronomers. The opening of the piece is mysterious and atmospheric, evoking the dark, cold environment of an interstellar cloud before the onset of star formation.

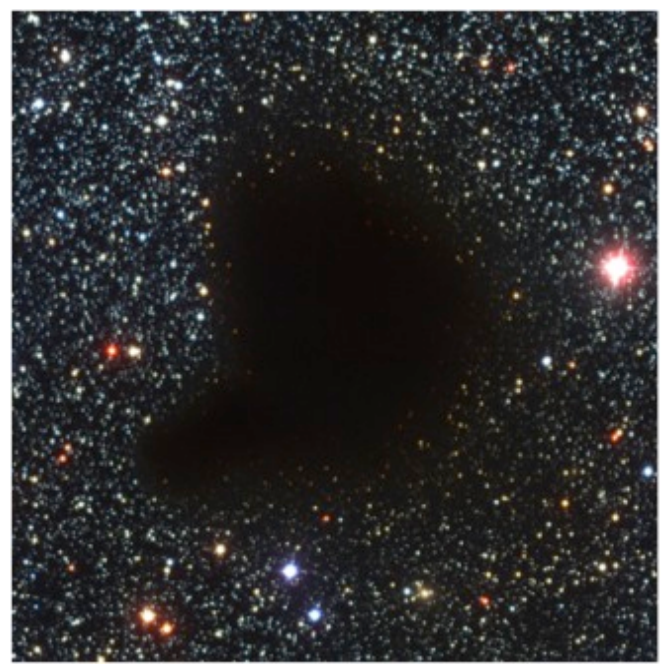

Figure 1. Nebulae - with newly formed stars. Image: NASA ESA Hubble Heritage Team (STScI, AURA ESA Hubble Collaboration)

2 Astronomy Camp: http://www.astronomycamp.org.

3 Project ASTRO is an educational outreach programme sponsored by the Astronomical Society of the Pacific that pairs astronomers with classroom teachers to enhance astronomy and science instruction. 
A fast, powerful passage suddenly ensues, representing the arrival of a supernova shock front - one of the possible mechanisms for triggering star formation. A large dance section, forming the centrepiece of the work, represents the collapse and fragmentation of the interstellar cloud.

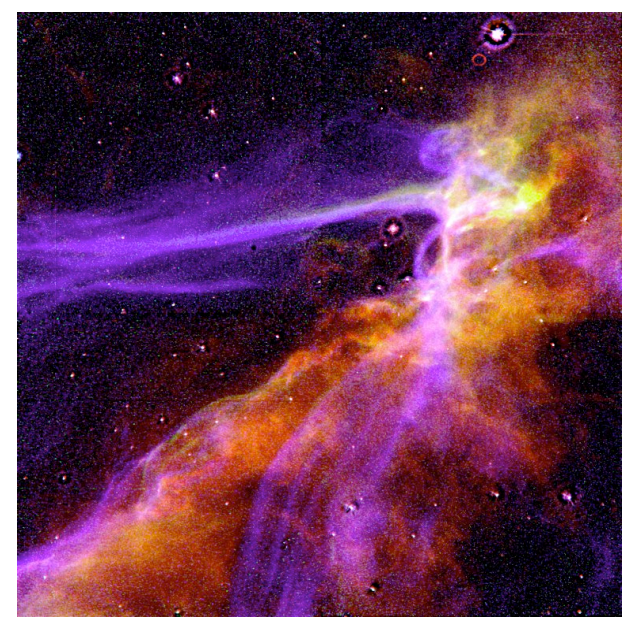

Figure 2. Nebulae - molecular cloud, 'Cygnus Loop Supernova Blast Wave', image: $\mathrm{ESO}^{4}$

Figure 3. Nebulae supernova shock front, 'Ring Around Supernova', image: NASA, courtesy of nasaimages.org. ${ }^{5}$

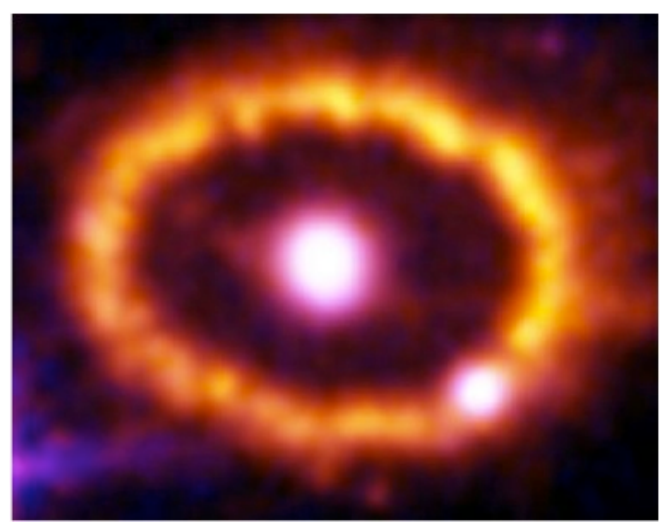

4http://www.dvidshub.net/image/837918/cygnus-loop-supernova-blastwave\#.UzIjm9x02Qw.

5 http://hubblesite.org/newscenter/archive/releases/2004/09/image/c/. 
As the dance unfolds, it grows in intensity and rhythmic complexity, depicting the protostar stage of star formation. Powerful chords signal the ignition of nuclear fusion, marking the birth of a star. Nebulae concludes with a dramatic, rapid toccata, reflecting both the brilliance of the newlyformed star and the grandeur of the nebula which gave it birth.

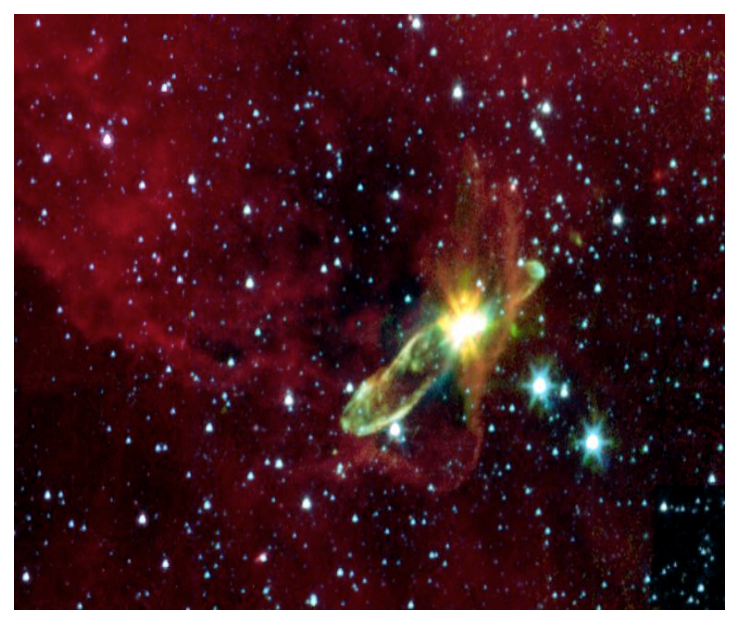

Figure 4. Nebulae Protostar, 'Young Star, Dark Cloud', Image: A. Noriega-Crespo (SSC Caltech) et al., JPL, NASA, courtesy of nasaimages.org. ${ }^{6}$

American composer Myron Roberts' Nova (1973) is an organ solo work with a length and scale similar to that of Nebulae. Unlike Nebulae, Nova is not directly narrative, though it does contain narrative elements. The work opens with a lengthy, unsettled introduction using many of the organ's various tone colours, calling to mind the increasing instability of a star before going supernova. This sense of instability increases via rapid and highly dissonant passages played on the full power of the organ. The work's central section comprises intense, rapidly repeating chords, and unfolds in a gradual buildup that culminates in a series of ferocious sonorities, depicting the supernova explosion itself. Nova's final section evokes the supernova remnant as it disperses into space. In this closing passage, the organ's highest and lowest tones are superimposed, providing an excellent example of the use of unusual tone colours in evoking astronomical phenomena.

At INSAPVII a venue featuring a pipe organ was unavailable. Nebulae and Nova were thus presented via CD recording and a highquality sound system. For both works, the author used a recording of his

6 http://apod.nasa.gov/apod/ap031226.html. 
own performance on the organ in Holsclaw Hall at The University of Arizona. In the conference presentation, both works were accompanied by projected images elucidating connections between the music and astronomical phenomena.

Nebulae and Nova have both been featured in live performances by the author. Nebulae has been performed by American organists in recitals throughout the United States, and was included in concerts by organist Frank Shelton (Colorado College) at Notre Dame Cathedral and St. Sulpice Church in Paris during October 2010. Nebulae and Nova have also been incorporated into education/outreach programmes, including a series of guided listening experiences developed by the author for The University of Arizona Astronomy Camp. In February 2010, the author gave a live performance of Nebulae at an astronomy/music outreach event at Biosphere 2, a research and education facility located just outside Tucson and operated by The University of Arizona. ${ }^{7}$ For this performance, a high-quality digital organ was installed.

Nebulae and Nova are excellent examples of the manner in which the tremendous sonic resources of the pipe organ can be used in the composition and performance of music inspired by astronomical phenomena. Subsequent compositions by the author include Pleiades Visions, a large-scale composition for solo organ inspired by indigenous music and mythology connected with the Pleiades star cluster. Pleiades Visions premiered in 2012. The author plans to continue composing new organ works with astronomical connections, and hopes to incorporate those works in innovative, interdisciplinary live performances.

7 Biosphere 2: http://www.b2science.org. 